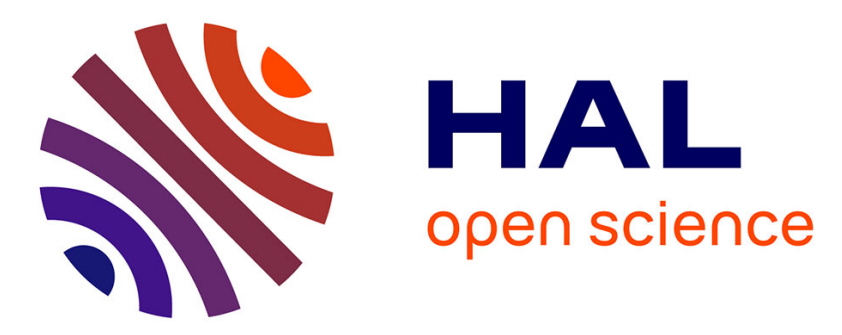

\title{
A numerical and experimental validation of the room acoustics diffusion theory inside long rooms
}

Chiara Visentin, Nicola Prodi, Vincent Valeau, Judicaël Picaut

\section{To cite this version:}

Chiara Visentin, Nicola Prodi, Vincent Valeau, Judicaël Picaut. A numerical and experimental validation of the room acoustics diffusion theory inside long rooms. 21st International Congress on Acoustics, Jun 2013, Montréal, Canada. 8p, ill., cartes, graphiques, bibliogr. hal-00845722

\section{HAL Id: hal-00845722 \\ https://hal.science/hal-00845722}

Submitted on 17 Jul 2013

HAL is a multi-disciplinary open access archive for the deposit and dissemination of scientific research documents, whether they are published or not. The documents may come from teaching and research institutions in France or abroad, or from public or private research centers.
L'archive ouverte pluridisciplinaire HAL, est destinée au dépôt et à la diffusion de documents scientifiques de niveau recherche, publiés ou non, émanant des établissements d'enseignement et de recherche français ou étrangers, des laboratoires publics ou privés. 


\section{INTRODUCTION}

The room-acoustics diffusion theory is a recently developed energetic model for the prediction of the sound field in urban streets and rooms with diffusely reflecting boundaries $[1,2]$. The model is based on the analogy between the propagation (diffusion) of particles inside a scattering medium and the propagation of the reverberant part of the sound field in rooms $[3,4]$. Following the analogy, the sound field can be described by means of a diffusion process and modeled as a cloud of sound particles propagating inside the room at the speed of sound $c$, carrying an infinitesimal amount of energy and undergoing reflections and absorption phenomena at the room boundaries. Accordingly, the analytical computation of the sound particles density at each receiver position returns the local sound energy density.

The numerical solution of the diffusion equation, together with appropriate boundaries conditions, allows the prediction of the sound field even in rooms with complex shapes, with good accuracy and limited computational resources [5]. Over the years, the original model has been further developed and applied to rooms of different shape [6, 7, 8, 9, 10,11, 12, 13, 14], showing a good agreement between the predicted and the measured (or simulated) values of sound pressure level and reverberation time inside the rooms.

Anyway these studies only provide an indirect validation of the diffusion theory, without dealing with the underlying equation of the model, expressing a proportionality relationship between sound intensity $\mathbf{I}(\mathbf{r})$ and gradient of sound energy density $w(\mathbf{r})$. This relationship, named "Fick's law of diffusion", is expressed through a diffusion gradient equation and states that an energy density gradient produces a motion of sound particles from a high density area to a low density one (energy flow):

$$
\mathbf{I}(\mathbf{r})=-D \nabla w(\mathbf{r})
$$

where $D$ is the diffusion coefficient. Following the analogy with the particles diffusion in a scattering medium, the diffusion coefficient is a constant for a given room and can be expressed as $D_{\text {th }}=\lambda c / 3 ; \lambda$ is the mean free path of the room and inside a room with diffusely reflecting boundaries depends on the room volume $V$ and the room surface $S$ as $\lambda=4 V / S$ [15].

As the proportionality relationship does not follow from the wave equation, it is important to assess the effective link between the energy density gradient and the acoustic intensity in order to understand the validity of the diffusion theory inside enclosures. This kind of analysis was performed in ref. [16], where the Fick's law in stationary state was numerically verified in long rooms with diffusely reflecting boundaries. In the study, a numerical approach able to simulate the real dynamics of a set of sound particles undergoing multiple reflections inside a room has been employed to predict the local values of energy density and sound intensity and then derive an estimate of the diffusion coefficient. The numerical analysis shows that inside long rooms the local value of the diffusion coefficient is not a constant but varies regularly, starting from the theoretical value (close to the source) and linearly increasing with the distance from the source. The rate of increase depends on the cross-sectional ratio of the room and the absorption properties of the boundaries.

An experimental approach to the direct validation of the diffusion theory is proposed in ref. [17] where the relationship between energy density gradient and sound intensity is investigated by means of measurements inside the scale model of a long room. A direct and experimental estimate of the local diffusion coefficient is provided, showing again an increasing trend of the diffusion coefficient along the length of the room. Additionally, the experimental study examines the possibility of describing the sound field in rooms with mixed (specular and diffuse) reflections through a diffusion process. The sensitivity of the diffusion coefficient to the reflection properties of the room walls is then assessed.

The present work aims to compare the local value of the diffusion coefficient derived by means of numerical simulations and experimental measurements, with reference to long 
enclosures and a sound field in stationary conditions. By varying the boundaries scattering coefficient, the analysis is extended to enclosures where mixed reflections take place. Firstly the main results of the measurements in the scaled long room are recalled, together with the experimental estimate of the local diffusion coefficient. Then the numerical model of the experimental set up is described and simulated and measured energetic quantities are compared. Finally a minimization procedure is carried out to perform a comparison between numerical and experimental estimate of the diffusion coefficient.

\section{EXPERIMENTAL ESTIMATE OF THE DIFFUSION COEFFICIENT}

For the experimental analysis of the relationship between reverberant energy density and sound intensity inside long rooms a 1:16 scale model was set up. The full-size (FS) equivalent dimensions of the model are $40 \times 8 \times 4 \mathrm{~m}^{3}$; a detailed description of the scale model features and the measurement set up can be found in ref. [17]. In order to assess the sensitivity of the diffusion coefficient to the reflection properties of the room boundaries, two different configurations were modeled: a long room with flat boundaries (characterized by purely specular reflections) and a long room prepared with scattering elements over the boundaries (characterized by mixed reflections). The scattering and absorption coefficients of the room walls (flat finishing and scattering frames) were assessed by means of measurements inside a scaled reverberation chamber [18].

The measurements inside the model were accomplished with a three dimensional sound intensity probe, in order to get a direct and coincident measure of the sound pressure and the three axial components of the particle velocity. Prior to the measurements the probe was calibrated with a set of digital filters, ensuring a flat response within the frequency range $1.5-18 \mathrm{kHz}$. The measurements were performed over 46 receiver positions with the sine-sweep technique; the elaboration of the four IRs derived for each position allowed the calculation of the local energy density and sound intensity in one-third octave bands. Moreover, around each main receiver position an additional set of six measurements was collected to be used for the estimate of the local energy density gradient. Starting from the measurements, the relationship between sound intensity and energy density gradient was then assessed through the analysis of the local diffusion coefficient, as:

$$
D_{\text {meas }}(\mathbf{r})=\frac{|\mathbf{I}(\mathbf{r})|}{|\nabla w(\mathbf{r})|} .
$$

The ratio between the measured diffusion coefficient and its theoretical value $D_{\text {th }}$ is showed in Fig. 1 for the one-third octave band of $500 \mathrm{~Hz}$ (FS). The results refer to the receivers located over the horizontal plane at $z=1.6 \mathrm{~m}$ (FS); consistently with the linear trend of the diffusion coefficient found in ref. [16] the linear regression of the data is reported too. The data show a great dispersion due to the difficulty of the experimental measurements and, especially, to the measurement of the energy density gradient. Nevertheless, relying on the regressions, a definite variation of the diffusion coefficient with the distance can be observed, influenced by the scattering coefficient of the room boundaries. Inside the room with flat boundaries the measured diffusion coefficient greatly increases over the distance, starting from a value of $5 D_{\text {th }}$ and sloping up to the value of $17 D_{\text {th }}$. Conversely, inside the room with diffusely reflecting boundaries a smaller variation is observed: starting from a value of $4 D_{\text {th }}$, at the end wall of the room the diffusion coefficient reaches the value of $6 D_{\text {th }}$. Increasing the amount of specular reflections inside the room leads to a raise in both initial value and slope of the measured diffusion coefficient. Then, inside long rooms is experimentally verified that the relationship between the energy density gradient and the sound intensity is not characterized by a constant diffusion coefficient. A definite increase from the source to the end wall of the room is observed, whose slope depends on the reflection properties of the room walls. 


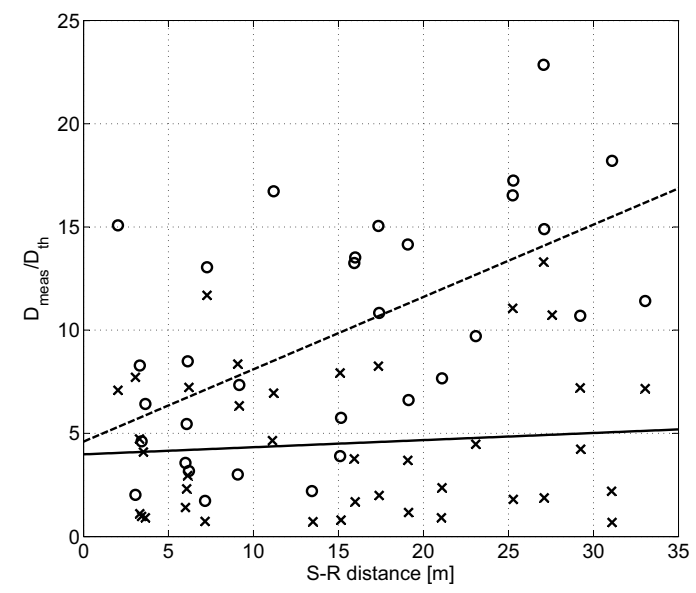

FIGURE 1: Ratio between the measured diffusion coefficient $\left(D_{\text {meas }}\right)$ and its theoretical value $\left(D_{\text {th }}\right)$ as a function of the distance from the source. Results for the one-third octave band of $500 \mathrm{~Hz}$ (FS): (o and dashed line) long room with flat boundaries, ( $\times$ and solid line) long room with diffusely reflecting boundaries.

The general trend of the results for the long room with diffusely reflecting boundaries is in agreement with that obtained by means of numerical simulations [16], predicting a linear increase of the diffusion coefficient with the distance from the source. Anyway, differences can be observed between the estimated initial values (equal to $D_{\text {th }}$ for the numerical simulations and bigger than $D_{\text {th }}$ for the measurements). These discrepancies could be related to the reflection properties of the room surfaces, being the numerical results only referred to long rooms characterized by purely diffuse reflections. Therefore, in order to perform a closer comparison between numerical and experimental estimate, numerical simulations of the two experimental set ups were carried out.

\section{NUMERICAL SIMULATION OF THE EXPERIMENTAL SET UP}

The same code employed for the previous numerical analysis [19] was used for the numerical simulations of the experimental set ups. It is based on the particle tracing approach: particles are emitted by a source and propagate at the speed of sound along straight lines, carrying an infinitesimal amount of energy and undergoing reflections and absorption phenomena at the room boundaries; the contribution of all particles are collected at the receiver positions in order to compute the energetic quantities. The code allows the management of the surface properties, through the choice of absorption $\alpha$ and scattering $s$ coefficients and the type of reflection law.

The sound field was numerically investigated inside the model of a $40 \times 8 \times 4 \times \mathrm{m}^{3}$ long room with an omnidirectional sound source located close the end wall, emitting a constant sound power level. Particle-tracing simulations were carried out with $N_{0}=5 \times 10^{6}$ particles on a total time $T=4 \mathrm{~s}$ and a time step $\Delta t=0.002 \mathrm{~s}$. The values $\alpha$ and $s$ were derived from the measurements inside the scaled reverberation chamber; the diffuse reflections were modeled according to Lambert's cosine law. One simulation was performed for each one-third octave band. The same source and receiver positions over the horizontal plane at $z=1.6 \mathrm{~m}$ as in the experimental set up were simulated and, in addition, the receivers grid was thickened obtaining a regular step of $1 \mathrm{~m}$ (FS). At each receiver the stationary energy density and intensity vector was obtained and used for the numerical estimate of the local diffusion coefficient $D_{\text {est }}$, according to Eq. (2). 


\section{Comparison of Numerical and Experimental Results}

The acoustical quantities resulting from the numerical simulations have been compared with those measured inside the scale models. The following comparisons refer to the long room with diffusely reflecting boundaries but similar conclusions can be drawn also for the long room with flat boundaries.

First, the spatial distribution of the normalized intensity vectors over the horizontal plane is considered. In Fig. 2 the numerical and experimental distributions are represented for the one-third octave band of $500 \mathrm{~Hz}$ (FS). From the experimental distribution, it can be noticed that while the energy flow presents a prevailing orientation along the $x$-direction, it is not completely mono-dimensional and small, random deviations from the main directions are always present. These local deviations are due to the energy recirculation phenomena characterizing the real sound field and are probably enhanced by small irregularities in the scale model set up. Conversely, the numerical code predicts a purely mono-dimensional behavior of the sound field, failing to model the whole three-dimensional distribution of the intensity vectors inside long rooms. The same remarks can be extended to the case of long room with flat boundaries, in spite of a general more one-dimensional distribution of the experimental energy flow (results not displayed in this paper).

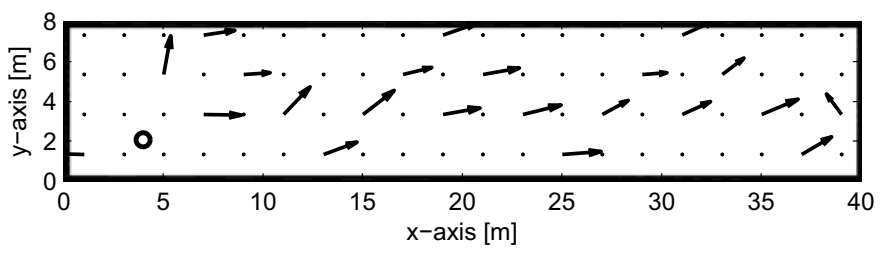

(A) Experimental intensity vectors

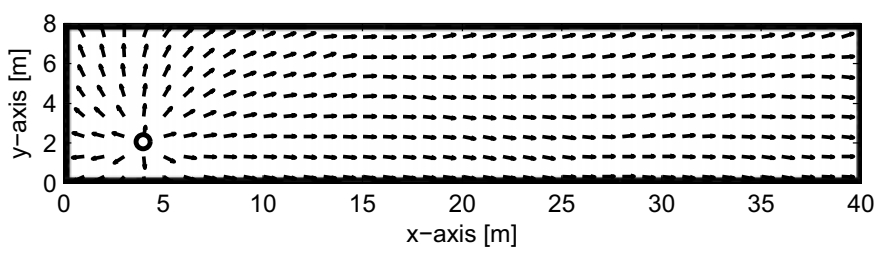

(B) Numerical intensity vectors

FIGURE 2: Spatial distribution of the intensity vectors over the horizontal plane at $z=1.6 \mathrm{~m}$. Normalized results for the scaled long room with diffusely reflecting boundaries and the one-third octave band of $500 \mathrm{~Hz}$ (FS).

It can be concluded that a direct comparison between experimental and numerical results can only be performed by taking into account the predominant component of the sound field. Therefore, the following comparisons between simulated and measured acoustical quantities are referred to the component of along the $x$-direction. In Fig. 3(a) the simulated and experimental spatial decays of the energy density level are represented, for the one-third octave band of $500 \mathrm{~Hz}$. A satisfactory agreement can be observed, with small differences increasing with the distance from the source and reaching $1.5 \mathrm{~dB}$ at the end wall of the room. Then, concerning the spatial decay of the sound intensity level (Fig. 3(b)), it can be noticed that the same trend characterizes the two set of data even though the numerical code provides a systematic overestimate of the measured data.

\section{LEAST MEAN SQUARE ESTIMATE OF THE DIFFUSION COEFFICIENT}

Following the results of the comparison between measured and numerical energetic quantities, a least mean square estimate procedure for the comparison of the diffusion 


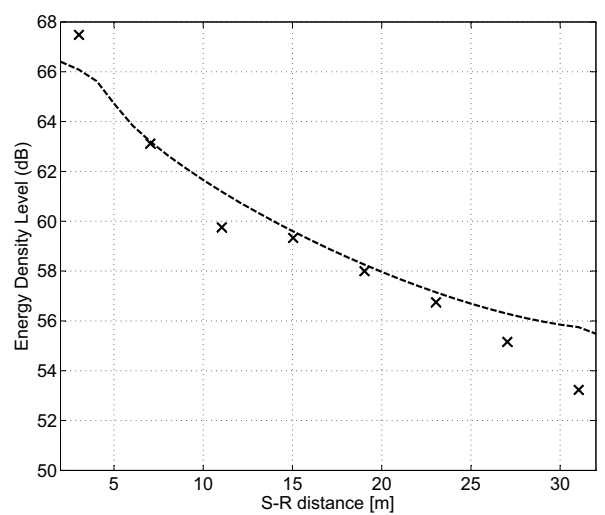

(A) Energy density level



(B) Sound intensity level

FIGURE 3: Energy density level and sound intensity level spatial decay inside the long room with diffusely reflecting boundaries. Results for the one-third octave band of $500 \mathrm{~Hz}$ (FS): ( $\times$ ) measured values, (dashed line) numerically simulated values.

coefficients was introduced. Indeed, the $D_{\text {meas }}$ values obtained from the measurements can not be directly employed for the comparison, being calculated in the hypothesis of a three-dimensional sound field. The least mean square estimate takes instead into account only the measured acoustic quantities component along the $x$-direction, allowing a meaningful comparison with the numerical simulations.

A preliminary hypothesis on the diffusion coefficient variation with the distance from the source is introduced, derived from the results of the numerical simulations: $D_{\text {meas }}$ is supposed to vary linearly with the distance from the source, following the general law $D_{\text {meas }}(\boldsymbol{r})=D_{\text {th }} \cdot(\hat{a}+\hat{b} r)$.

The least mean square estimate procedure follows three main steps:

1. Starting from the measured values of the sound energy density, a regression law $\hat{w}(\boldsymbol{r})=f(\boldsymbol{r})$ is calculated, to describe the spatial decay along the line from the source to the end wall. For each frequency band a best fit procedure allows to obtain the coefficients of the regression curve, expressed as a power law for the room with flat boundaries and as an exponential law for the room with diffusely reflecting boundaries. Different regression laws were chosen for the two configurations as a consequence of the different sound fields established in the models; in both cases the law maximizing the correlation coefficient for the majority of the frequency bands, was chosen.

2. A theoretical regression curve is calculated, in order to describe the spatial decay of the sound intensity, according to the diffusion gradient equation and the hypothesis of a linear variation of the diffusion coefficient. The regression law can be expressed as: $\hat{I}(\boldsymbol{r})=-D_{\mathrm{th}}(\hat{a}+\hat{b} r) f^{\prime}(\boldsymbol{r})$, where the values of $\hat{a}$ and $\hat{b}$ are the only unknown quantities and $f^{\prime}(\boldsymbol{r})$ expresses the spatial derivative of the regression law obtained in step 1.

3. The parameters $\hat{a}$ and $\hat{b}$ are estimated with a least mean square procedure, comparing the regression curve of step 2 with the $I_{x}(\boldsymbol{r})$ values measured inside the scale model. In particular, the solution of the estimate problem is obtained employing an empirical trial method, that is graphically investigating the quadratic error $E(\hat{a}, \hat{b})$ over a defined range of variation of the estimate coefficients $\hat{a}$ (intercept) and $\hat{b}$ (slope). The quadratic error is 
calculated as:

$$
E(\hat{a}, \hat{b})=\sum_{i=1}^{N_{p}}\left(I_{x, i}-\hat{I}_{i}\right)^{2},
$$

where $N_{p}$ is the number of considered receiver points.

In Fig. 4 a graphical comparison between numerical and least mean square estimate of the diffusion coefficient is presented for the one-third octave band of $500 \mathrm{~Hz}$ (FS).

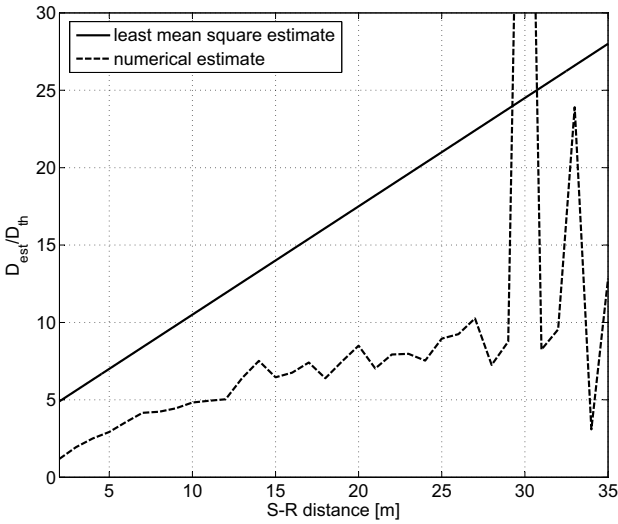

(A) Room with flat boundaries

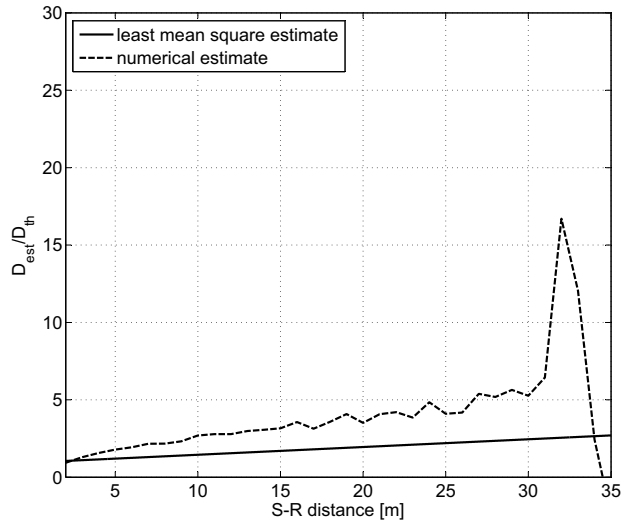

(B) Room with diffusely reflecting boundaries

FigURE 4: Ratio between the estimated diffusion coefficient $\left(D_{\text {est }}\right)$ and its theoretical value $\left(D_{\text {th }}\right)$ as a function of the distance from the source. Results for the one-third octave band of $500 \mathrm{~Hz}$ (FS): (dashed line) numerical estimate, (solid line) least mean square estimate.

Concerning the numerical estimate, it can be seen that $D_{\text {est }}$ increases linearly with the distance from the source, starting from the theoretical value. The rapid fluctuations taking place in the region close to the end wall are believed to be related to numerical errors in the computation of $D_{\text {est }}$ (as discussed in ref. [16]). According to these results, the boundaries scattering coefficient only influences the rate of increase of the diffusion coefficient, without affecting its value close to the source, always equal to $D_{\text {th }}$. In the long room with flat boundaries it is observed an increasing rate of $D_{\text {est }}$ five times bigger than in the room with diffusely reflecting boundaries.

As regards the least mean square estimate, the same general trend discussed about $D_{\text {meas }}$ is verified: the value of the boundaries scattering coefficient influences both slope and initial value of the diffusion coefficient. Inside the room with flat boundaries the diffusion coefficient increases greatly with the distance from the source, varying from $5 D_{\text {th }}$ up to $28 D_{\text {th }}$. On the contrary, inside the room with diffusely reflecting boundaries, the estimated diffusion coefficient shows a weakly increasing trend, sloping up from $D_{\text {th }}$ to $3 D_{\text {th }}$.

The comparison between numerical and least mean square estimate shows that although a good agreement is found in terms of trend of variation and sensitivity to the boundaries scattering coefficient, still several discrepancies can be observed. Both approaches validate the hypothesis that for long rooms the relationship between sound intensity and energy density gradient is not properly represented by a constant diffusion coefficient. $D_{\text {est }}$ varies inside the room, increasing from the source to the end wall; its estimated (or measured) values are always bigger than the theoretical, constant value. The rate of increase of $D$ is governed by the scattering coefficient of the boundaries: a weak increase is observed when the scattering coefficient is close to one (and the hypothesis of the diffusion theory are fulfilled) whereas, conversely, decreasing the amount of specular reflections inside the room leads to a great variation of $D$ with the distance. Anyway, the comparison between numerical and least mean 
square estimate shows that in the long room with flat boundaries the numerical code leads to an underestimate of the diffusion coefficient, both in slope and initial value. In the long room with diffusely reflecting boundaries both approaches yield to the same initial value $D_{\text {th }}$ but the numerical code provides an overestimate of the diffusion coefficient slope.

\section{Conclusions}

In this work the effective relationship between intensity and energy density gradient inside long rooms in stationary state is investigated by means of numerical simulations and experimental measurements. Measurements of sound pressure and particle velocity were performed inside a scaled long room and used to calculate the local values of energy density and sound intensity, whose ratio expresses the diffusion coefficient $D$. Two configurations with different surface finishing were set up in order to analyze the sensitivity of the diffusion coefficient to the amount of specular reflections inside the room. Numerical simulations of the experimental configurations were carried out with a particle tracing code. The comparison between simulated and measured acoustic quantities shows that the numerical code predicts a purely mono-dimensional energy flow inside the room whereas the measurements highlight small, random deviation from the main direction due to energy recirculation phenomena. Then, a least mean square procedure, based on a minimization algorithm, is proposed to compare numerical and experimental estimate of the diffusion coefficient.

Both approaches return the same general trend, showing that inside long rooms the diffusion coefficient is no longer a constant but linearly increases along the length of the room. Inside an elongated room it is then verified that the reverberant sound field is described by non-homogeneous diffusion and characterized by a spatially varying diffusion coefficient. Slope and initial value of the diffusion coefficient are influenced by the properties of the room boundaries: increasing the amount of specular reflections inside the room, affects both initial value and slope of $D$.

It can be concluded that the model of homogeneous diffusion can not properly represent the real physics of the sound field inside long rooms. Further experimental investigations on the relationship between energy density and sound intensity are believed to be necessary, especially inside non-proportionate enclosures. The equation underlying the theoretical model should be also reconsidered, restarting from the transport equation, in order to properly represent the sound propagation inside enclosures, also considering the reflection properties of the room boundaries.

\section{ACKNOWLEDGMENTS}

The authors would like to thank Microflow Technologies for loaning the sound intensity probe used in the scale model measurements.

\section{REFERENCES}

[1] J. Picaut, L. Simon, and J. Polack, "A mathematical model of diffuse sound field based on a diffusion equation”, Acust. Acta Acust. 83, 614-621 (1997).

[2] J. Picaut, L. Simon, and J. Polack, "Sound field in long rooms with diffusely reflecting boundaries”, Appl. Acoust. 56, 217-240 (1999).

[3] F. Ollendorff, "Statistical room-acoustics as a problem of diffusion (a proposal)", Acustica 21, 236-245 (1969). 
[4] P. Morse and H. Feshbach, Methods of theoretical physics (McGraw-Hill, New York, 1953).

[5] V. Valeau, M. Hodgson, and J. Picaut, "A diffusion-based analogy for the prediction of sound fields in fitted rooms”, Acust. Acta Acust. 93, 94-105 (2007).

[6] A. Billon, V. Valeau, A. Sakout, and J. Picaut, "On the use of a diffusion model for acoustically coupled rooms”, J. Acoust. Soc. Am. 120, 2043-2054 (2006).

[7] Y. Jing and N. Xiang, "A modified diffusion equation for room-acoustic predication", J. Acoust. Soc. Am. 121, 3284-3287 (2007).

[8] A. Billon, J. Picaut, and A. Sakout, "Prediction of the reverberation time in high absorbent room using a modified-diffusion model”, Appl. Acoust. 69, 68-74 (2008).

[9] Y. Jing and N. Xiang, "On boundary conditions for the diffusion equation in room-acoustic prediction: Theory, simulations, and experiments”, J. Acoust. Soc. Am. 123, 145-153 (2008).

[10] A. Billon, C. Foy, J. Picaut, V. Valeau, and A. Sakout, "Modeling the sound transmission between rooms coupled through partition walls by using a diffusion model.", J. Acoust. Soc. Am. 123, 4261-71 (2008).

[11] A. Billon, J. Picaut, C. Foy, V. Valeau, and A. Sakout, "Introducing atmospheric attenuation within a diffusion model for room-acoustic predictions (L)”, J. Acoust. Soc. Am. 123, 4040-4043 (2008).

[12] N. Xiang, Y. Jing, and A. Bockman, "Investigation of acoustically coupled enclosures using a diffusion-equation model”, J. Acoust. Soc. Am. 126, 1187-1198 (2009).

[13] C. Foy, V. Valeau, A. Billion, J. Picaut, and A. Sakout, "An empirical diffusion model for acoustic prediction in rooms with mixed diffuse and specular reflections", Acust. Acta Acust. 95, 97-105 (2009).

[14] J. Escolano, J. Navarro, and J. Lopez, "On the limitation of a diffusion equation model for acoustic predictions of rooms with homogeneous dimensions (L)", J. Acoust. Soc. Am. 128, 1586-1589 (2010).

[15] H. Kuttruff, Room acoustics, 4th edition (Spon Press, London, 1999).

[16] C. Visentin, N. Prodi, V. Valeau, and J. Picaut, "A numerical investigation of the Fick's law of diffusion in room acoustics”, J. Acoust. Soc. Am. 132, 3180-3189 (2012).

[17] C. Visentin, N. Prodi, V. Valeau, and J. Picaut, "An experimental analysis of the relationship between reverberatn acoustic intensity and energy density inside long rooms", in INTERNOISE2012 Conference Proceedings, New York (August 19-22, 2012).

[18] C. Visentin, "A numerical and experimental validation of the diffusion equation applied to room acoustics", PhD thesis, University of Ferrara, Italy (2012).

[19] I-simpa web site, http://i-simpa.ifsttar.fr/. 